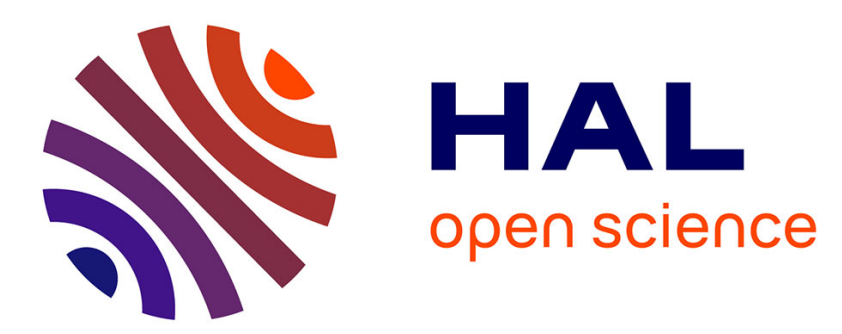

\title{
Recherches récentes sur les carrières antiques de Gaule: bilan et perspectives
}

\author{
Jean-Claude Bessac, Robert Sablayrolles
}

\section{To cite this version:}

Jean-Claude Bessac, Robert Sablayrolles. Recherches récentes sur les carrières antiques de Gaule: bilan et perspectives. Gallia - Archéologie de la France antique, 2002, Carrières antiques de la Gaule, 59, pp.175-188. 10.3406/galia.2002.3104 . hal-01912535

\section{HAL Id: hal-01912535 \\ https://hal.science/hal-01912535}

Submitted on 20 Jan 2020

HAL is a multi-disciplinary open access archive for the deposit and dissemination of scientific research documents, whether they are published or not. The documents may come from teaching and research institutions in France or abroad, or from public or private research centers.
L'archive ouverte pluridisciplinaire HAL, est destinée au dépôt et à la diffusion de documents scientifiques de niveau recherche, publiés ou non, émanant des établissements d'enseignement et de recherche français ou étrangers, des laboratoires publics ou privés.

\section{(ㅇ)(1) $\$$}

Distributed under a Creative Commons Attribution - NonCommercial - NoDerivatives| 4.0 


\title{
RECHERCHES RÉCENTES
}

\section{SUR LES CARRIÈRES ANTIQUES DE GAULE}

\section{Bilan et perspectives}

\author{
Jean-Claude Bessac et Robert Sablayrolles
}

\section{LA RECHERCHE ET SON ÉVOLUTION}

L'introduction de ce dossier en faisait déjà état: l'avancement de la recherche sur les carrières antiques de la Gaule n'est pas encore mûr pour autoriser une vé yitable synthèse. En l'état actuel des données disponitges, dont les diverses contributions rassemblées ici donnent une bonne image, il faut se contenter d'un pollh: sur la question, qui insiste sur les apports propres a ay houvelles études, sur les hypothèses qu'ils suscitent et -ir les perspectives de recherche ${ }^{107}$. Les secteurs où les cunaissances n'ont que peu ou pas évolué seront évgqués seulement de façon succincte. L'activité archéolog que de ces trente dernières années dans les carrières antiques de Gaule s'est concrétisée de diverses manières, depuis les prospections et les inventaires de productions jusqu'à la fouille stratigraphique, pour laquelle le site de Saint-Boil, en 1972, constitua une entreprise pionnière (cf. Monthel, supra, p. 89-120). C'est désormais à plus d'une vingtaine d'opérations de ce type que s'élève le bilan dans ce secteur de la recherche archéologique nationale. Toutes n'ont pas eu la même ampleur, mais leur diversité géographique et géologique donne néanmoins une meilleure vision de cette activité économique antique, souvent très liée à la construction, et

107. En dehors des recherches qu'ils ont personnellement dirigées, les auteurs de la présente synthèse ont visité la majorité des sites présentés dans le dossier et ont parfois contribué à la mise en route scientifique de quelques opérations sur le terrain (carrières de La Lic, de La CroixGuillaume et du Tendu) ou expertisé des vestiges dans d'autres exploitations (Locuon et Saint-Boil); les commentaires sur ce bilan ne peuvent donc être considérés comme le point de vue d'observateurs totalement extérieurs à ces recherches. qui s'est particulièrement développée durant la période romaine.

Jusqu'en 1972, la fouille de carrières n'avait guère concerné, en Europe occidentale, que la région rhénane en Allemagne (cf. Lukas, supra, p. 155-174). Aujourd'hui, une meilleure répartition géographique des résultats archéologiques est acquise, du moins en France. Cependant, malgré le mérite des archéologues qui se sont attelés à de telles tâches, la carte des carrières étudiées ne reflète encore que la répartition des spécialistes de cette question, et non l'implantation réelle des exploitations antiques. La plupart des villes romaines, même secondaires, ont eu leurs chantiers d'extraction, souvent multiples et offrant diverses qualités de pierre, dans la mesure où ces cités se trouvaient à proximité d'affleurements rocheux. Ce n'est donc qu'un pourcentage très faible de l'ensemble des exploitations qui a été partiellement étudié sur le terrain et qui est présenté ici. En dehors des opérations proprement archéologiques, beaucoup d'autres carrières antiques, ou supposées telles, ont fait l'objet de notes ou d'articles, sans que leurs auteurs aient recouru à des investigations particulières sur le terrain. Même si ces données ne sont pas présentées ici, leur apport est néanmoins pris en compte, dans la mesure où il est bien étayé ou confirmé par les résultats de fouilles dans d'autres sites. Notons aussi l'absence d'une contribution promise sur les carrières antiques du cap Couronne près de Marseille: les auteurs des premières études sur le site ont finalement préféré attendre les résultats de nouvelles investigations en cours, plutôt que de proposer des informations qu'ils estimaient dépassées. 
À la lecture des résultats archéologiques obtenus ces dernières décennies en la matière, deux phases se distinguent nettement. La première, entre 1975 et 1990, avait surtout un caractère de recherche fondamentale, orientée par des problématiques scientifiques, sans trop d'entraves matérielles et administratives. Les aspects méthodologiques et les propositions typologiques ont connu alors un premicr développement, alimenté en sites inédits par quelques prospections thématiques et par des recherches de diffusion de la production, à l'exemple de celles du Bois des Lens et des bassins marbriers pyrénéens (cf. Bessac, supra, p. 29-51 et Fabre, Sablayrolles, supra, p. 61-81). Cet aspect initial de la recherche ne semble plus que marginal à présent, en particulier pour les prospections. Depuis une dizaine d'années, les archéologues s'orientent plutôt vers une recherche appliquée, qui s'exerce à la faveur de sauvetages urgents et surtout à l'occasion d'aménagements de sites d'intérêt culturel et touristique. Les chantiers archéologiques ouverts récemment dans les carrières antiques appartiennent essentiellement à cette dernière catégorie. Telle est la situation de la carrière de La Lie, près de Mâcon (cf. Cognot, supra, p. 121-131), mais aussi, et surtout, du chantier de L'Estel au Pont du Gard, qui profite de la dynamique du projet de l'aménagement général du site (cf. Bessac, Vacca-Goutoulli, supra, p. 11-28). Il est vaai qu'aux difficultés matérielles et à l'austérité notoire des fouilles en carrière se sont ajoutées peu à peu des lourdeurs administratives et des responsabilités, qui ne peuvent être surmontées et supportées que par de solides associations ou des institutions spécifiques, et non par des chercheurs plus ou moins isolés, comme c'était souvent le cas précédemment. À travers cette nouvelle orientation, qui est plus subie que voulue par les archéologues, s'esquisse une disparition relative des objectifs scientifiques de la recherche archéologique fondamentale programmée. L'intégration des carrières antiques dans le patrimoine monumental et touristique offre néanmoins d'importantes contreparties, susceptibles de profiter à la recherche. Des responsables de collectivités publiques, qui n'ont longtemps vu dans les lieux d'extraction abandonnés que de grands trous faciles à utiliser en dépotoirs, commencent à se sensibiliser à leur aspect monumental et ne sont plus opposés, comme auparavant, à leur étude archéologique et à leur conservation. D'un autre côté, les aménageurs de sites touristiques ont pris conscience du caractère monu- mental et historique des carrières antiques, et aussi de l'intérêt des visiteurs pour ces vestiges techniques.

Peutêtre plus que dans d'autres secteurs de l'archéologie, la recherche dans les carrières antiques a donc dû s'adapter à ces récentes orientations, indépendantes de la problématique scientifique. Parmi les objectifs mis en place par le Conseil supérieur de la recherche archéologique ces vingt dernières années figuraient la mise au point d'une méthodologie des techniques d'extraction et l'inventaire systématique des exploitations antiques. Le premier objectif, même s'il reste encore à développer, a bénéficié d'apports appréciables, qui constituent aujourd'hui une base de références reconnue, quoique encore lacunaire. La prospection systématique des carrières antiques n'a pas connu, en revanche, le développement que l'on attendait, mis à part les investigations menées dans les vallées des Pyrénées centrales et l'opération relativement modeste du Bois des Lens dans le Sud-Est (cf. Fabre, Sablayrolles, supra, p. 61-81 et Bessac, supra, p. 29-51). La prospection reste donc un point faible, et l'on manque aujourd'hui d'une véritable vue statistique sur le nombre et le volume des carrières antiques de Gaule. En revanche, les identifications géologiques d'affleurements de roches exploités par les Romains commencent à s'étoffer grâce à l'analyse des pierres des monuments. Les recherches conduites en ce sens sur les granites bretons sont, en la matière, exemplaires, d'autant qu'elles se doublent désormais d'une fouille en carrière dans la région de Carhaix.

\section{LA GRANDE DIVERSITÉ ARCHÉOLOGIQUE DES OPÉRATIONS}

Le bilan des recherches présenté ici porte sur une dizaine d'études, dont une est consacrée à l'évaluation des riches potentialités recélées par les sites d'extraction de Rhénanic et du nord-est de la Gaule (fin du I ${ }^{\mathrm{cr}} \mathrm{s}$. avant notre ère-VII" s., cf. Lukas, supra, p. 155-174). Parmi ces études, il existe une grande diversité de situations archéologiques. La plus ancienne opération est la fouille de la carrière de Saint-Boil (fin ${ }^{\text {er }}$ s. avant notre èreVII" s., cf. Monthcl, supra, p. 89-120) ; elle a commencé d'abord par un sauvetage urgent, puis s'est rapidement transforméc en fouille programméc, qui s'est terminéc en 1986. Les investigations dans l'affleurement du Bois des Lens (Protohistoire-Moyen Âge) sont parties de 
prospections générales et thématiques, commencées en 1974, pour déboucher sur une longue série de fouilles programmées et de sondages archéologiques, qui s'est achevée en 1996 (cf. Bessac, supra, p. 29-51). Les études des carrières de marbre des Pyrénées centrales (fin $I^{\mathrm{cr}} \mathrm{s}$. avant notre ère-Moyen Âge) ont débuté, dès 1991, par des prospections-inventaires, suivies de prospections thématiques et de sondages d'évaluation, de 1994 à 1999 (cf. Fabre, Sablayrolles, supra, p. 61-81). La recherche s'est désormais orientée vers l'étude de la diffusion des matériaux et des produits, accompagnée de travaux archéométriques sur les critères d'identification. À proximité de Lourdes, des découvertes fortuites, suivies de prospections thématiques et de sondages d'évaluation, ont révélé une intense exploitation du massif calcaire du Béout pour la production, du $\mathrm{V}^{\mathrm{e}}$ au VII' ${ }^{\mathrm{c}}$ s. de notre ère, de sarcophages bien attestés sur les sites archéologiques de la proche région, dans un rayon de 30 à $40 \mathrm{~km}$ (cf. Boudartchouk, supra, p. 53-60). Les fouilles en cours dans les carrières de La Lie ( ${ }^{\text {er }}$ s.époque moderne), près de Mâcon, engagées en 1994, sont la conséquence indirecte de la mise en valeur du site naturel par les collectivités et les associations locales (cf. Cognot, supra, p. 121-131). Il en va de même des fouilles entreprises en 1998 dans la carrière de L'Estel (milieu I ${ }^{\text {er }}$ s.), au Pont du Gard, qui se poursuivent actuellement dans le cadre d'une programmation trisannuelle (cf. Bessac, Vacca-Goutoulli, supra, p. 11-28). En Bretagne, la fouille de la carrière de granite* de Locuon (Haut-Empire), qui a débuté en 2000 , connaît, sur le site, une situation proche des précédentes, mais cette étude s'insère dans un processus de recherches plus systématiques sur les matériaux d'une cité antique, programme engagé dès 1995 (cf. Maligorne, Éveillard, Chauris, supra, p. 133-143). L'opération ponctuelle du site du Tendu (première moitié $d u I^{\text {er }} \mathrm{s}$.), près d'Argentomagus, est née d'un sauvetage urgent en 1994 et se singularise plus par les questions d'acheminement de la pierre par voie fluviale que par les techniques d'extraction (cf. Pichon, supra, p. 83-88). Enfin, les petites carrières de La Croix-Guillaume (II ${ }^{\mathrm{c}}-\mathrm{III}^{\mathrm{c}} \mathrm{s}$.), qui sont installées sur un sommet vosgien, ont été étudiées entre 1994 et 1999 en marge de la fouille d'une nécropole et d'un site d'habitat (cf. Heckenbenner, Meyer, supra, p. 145-154). Ces diverses opérations, objets des contributions ici réunies, présentent donc, pour l'essentiel, des conditions de recherche et des problématiques très différentes et, en général, complémentaires, ce qui constitue plutôt un avantage dans l'état actuel d'avancement de la recherche dans cette discipline.

\section{LES ROCHES}

On peut affirmer que, des plus tendres aux plus dures, toutes les roches susceptibles de donner des pierres dimensionnelles ont été exploitées durant l'Antiquité en Gaule: depuis les porphyres de l'Estérel, en passant par le basalte du Massif central, le granite armoricain, les marbres des Pyrénées, les grès de l'Est, jusqu'aux calcaires durs et tendres des grands bassins du Tertiaire (région parisienne, Nord, Bourgogne, façade ouest et bordure méditerranéenne). Toutes ces pierres sont attestées dans les monuments antiques, mais les fouilles archéologiques récentes de carrières n'ont concerné que quelques-unes de ces nombreuses variétés de roches.

Les calcaires tendres et fermes arrivent en tête des études avec les carrières de Bourgogne (Saint-Boil et La Lie), celles du Centre (site du Tendu), celles de la basse vallée du Rhône (Estel et Bois des Iens) et du SudOuest (Lourdes). Ce sont les variétés de pierre les plus répandues, il est donc statistiquement normal qu'elles figurent parmi les plus étudiées. Bien que les caractéristiques générales de ces calcaires, notamment leur résistance et leur finesse de grain, diffèrent sensiblement d'un ensemble à un autre, c'est le seul groupe de carrières qui offre la possibilité de comparaisons. Pour les autres catégories de matériaux, en effet, on ne recense qu'une opération par type de pierre. Le grès des Vosges, dans les carrières de La Croix-Guillaume, appartient à une catégorie ferme de couleur rose, très représentative des possibilités du massif. C'est le seul chantier d'extraction du grès étudié en Gaule, où cette roche affleure pourtant en différentes régions, notamment en Bourgogne et à l'extrémité occidentale des Pyrénées. Il est possible d'effectuer quelques rapprochements avec d'autres exploitations antiques de grès du Nord-Est et de Rhénanie, où ce matériau est également très présent, mais les observations effectuées dans ces derniers sites ne résultent pas de fouilles stratigraphiques.

Mis à part les travaux anciens de B. Sapène (1946), qui s'apparentaient plutôt à du sauvetage, les exploitations de marbre des Pyrénées n'ont bénéficié récemment 
que de modestes sondages et surtout de prospections, mais leurs productions et la diffusion de celles-ci ont fait l'objet d'études plus développées. L'inventaire des carrières antiques dans cette région est désormais mieux assuré : la prospection a révélé quelques sites nouveaux et l'application de critères rigoureux en matière de chronologie a fait disparaître quelques carrières considérées abusivement comme antiques dans les écrits anciens. L'absence de fouille programmée s'explique par l'intense utilisation, dans la longue durée, de la plupart des carrières: réexploitations modernes et contemporaines ont éradiqué ou enseveli sous des tonnes de déchets les vestiges antiques. L'environnement montagnard, qui rend certains sites difficilement accessibles et même dangereux, explique également cette lacune (cf. Fabre, Sablayrolles, supra, p. 61-81).

Après avoir été très longtemps totalement délaissées par les archéologues, les carrières de granite connaissent actuellement leurs premières fouilles en Gaule, grâce à l'intervention d'une équipe pluridisciplinaire sur le site de Locuon, près de Carhaix. Il s'agit d'une variété de granite parmi les moins dures et l'on ne peut donc transposer les résultats obtenus sur ce site sans tenir compte de cette particularité. En matière de provenance des pierres de taille des monuments antiques, les investigations sur les granits*, pris au sens général du terme, s'étendent à l'ensemble de la Bretagne.

\section{DES STRUCTURES D'EXPLOITATION TRÈS HÉTÉROGÈNES}

L'échantillonnage de carrières proposé dans le présent dossier souligne une diversité considérable dans les structures des exploitations. En premier lieu, en matière juridique, aucune carrière impériale, au sens propre du terme, n'a été identifiée. Aucune des exploitations de Gaule, et même de Rhénanie, n'est comparable, de fait, à celles d'Afrique du Nord ou du ProcheOrient, qui ont fourni des pierres ornementales de haute qualité (marbres, porphyres, granits, etc.), diffusées dans l'ensemble du monde romain (Gnoli, 1971). Même les exploitations de marbres pyrénéens paraissent, à la lumière des investigations récentes, ne pas appartenir à cette catégorie. Toutes les carrières impériales de marbre ont, en effet, leur production largement représentée dans les lieux de stockage à Rome ou aux abords de ses accès maritimes (Pensabene, 1994); or, les marbres des Pyrénées semblent plus que discrets en ces lieux. Les grandes carrières impériales, par ailleurs, font l'objet de mentions relativement fréquentes dans les textes antiques, alors que les références aux exploitations gauloises demeurent exceptionnelles (Bedon, 2000, p. 57-59).

Sur les fronts et les blocs des grandes carrières de roches ornementales d'Afrique du Nord et du Levant, la propriété des exploitations ne fait aucun doute puisqu'elle est bien attestéc par de multiples témoignages épigraphiques (Bruzza, 1870; Dubois, 1908; Fant, 1989, p. 49-254; Pensabene, 1994, p. 53-332). Aucune mention similaire n'a été découverte sur des roches ornementales de Gaule, y compris sur celles des Pyrénées, où, cependant, sont attestées des commandes impériales d'importance, comme le trophée de SaintBertrand-de-Comminges, réalisé dans un marbre de Saint-Béat parfaitement identifié (cf. Fabre, Sablayrolles, supra, p. 61-81). L'évaluation de la diffusion des marbres pyrénéens, que la difficulté de caractérisations fiables rend délicate, n'en est qu'à ses débuts. Bien représentés dans l'ensemble du bassin de la Garonne, ils paraissent plus rares ailleurs, même si leur diffusion au sud des Pyrénées est incontestable et même si quelques fragments de calcaire métamorphisé noir et blanc d'Aubert ont été recensés à Venise ou à Rome. L'éloignement des grands axes de communication, le climat rude de l'hiver pyrénéen et la fragilité de certains marbres ne désignaient pas ces carrières à l'attention de l'administration impériale. Leur développement est plutôt à expliquer par la recherche, dans un Sud-Ouest plus difficile à approvisionner en produits de luxe venus de la Méditerranée, d'un produit de substitution, qui intéressait plus les cités et les aristocraties locales que l'administration impériale. L'hypothèse de la mauvaise rentabilité de ces marbres n'est pas à écarter, mais ce n'est sans doute pas la seule raison du désintérêt apparent de la puissance impériale. D'autres carrières, même si elles ne produisaient pas des marbres ou des pierres marbrières, ont connu une importance commerciale, dont témoigne une large diffusion, dans un rayon bien supérieur à une centaine de kilomètres, telle la pierre des Lens, et aucun indice ne permet d'attribuer ces exploitations au domaine impérial.

Les documents épigraphiques du Nord-Est et de la Rhénanie, évoqués dans la contribution de D. Lukas 
(p. 155-174), révèlent sans aucun doute une intervention de détachements légionnaires dans l'exploitation des carrières de grès. Ils n'en constituent naturellement pas pour autant des titres de propriété, ni des légions ni de l'administration impériale. Les grès de l'Est, en effet, sont des pierres très communes, que l'on trouve en abondance et qui n'acquièrent un peu de valeur qu'à partir du moment où elles sont extraites. $\mathrm{Si}$, en marge de la fourniture de leurs propres besoins, les légions établies dans ces contrées tiraient quelques profits de ces exploitations, c'était de la même manière que les autres carriers, grâce à la plus-value de leur travail d'extraction, et non à cause d'une valeur propre du matériau. Les légions bénéficiaient certainement d'une concession temporaire pour l'extraction (Bedon, 1984, p. 173-175), et non d'un droit permanent de propriété. L'extension de ce rôle de l'armée à la plupart des affleurements de pierres de taille du Sud-Est (Bedon, 1984, p. 155) reste très hypothétique, pour ne pas dire abusive, tant que les indices épigraphiques feront défaut dans ces exploitations. Pourquoi de telles inscriptions se trouveraient-elles en quantité au Nord-Est et seraient absentes dans le Sud ? Par ailleurs, si la présence légionnaire n'avait rien que de très naturel aux abords du limes rhénan, elle s'expliquerait plus difficilement dans la Narbonnaise sénatoriale, éloignée de tout campement militaire des légions.

Un argument supplémentaire en faveur d'une concession ordinaire attribuée aux légions du Rhin est la présence dans les mêmes régions, surtout après le $\mathrm{I}^{\mathrm{er}} \mathrm{s}$., de nombreuses petites exploitations, que l'on peut qualifier d'artisanales, pour ne pas dire individuelles. L'exemple du site de La Croix-Guillaume illustre très bien cet autre aspect de l'exploitation du grès dans le Nord-Est. Même si l'on prend en compte les nombreux autres petits chantiers d'extraction antique dispersés sur les sommets vosgiens du voisinage, le volume extrait ici est très modeste et ne s'explique que dans le cadre d'une activité d'artisans polyvalents, à la fois carriers, tailleurs de pierre et sculpteurs. Ceux-ci devaient probablement assumer seuls, ou avec un aide, l'intégralité de la chaîne de production. Leurs excavations ne sont d'ailleurs jamais très profondes et ils s'attaquent de préférence aux couches supérieures des affleurements, plus aisées à extraire en raison de la grande fréquence des joints de stratification et des fissures, qui réduisent le travail de creusement. Les carrières exploitées par les légions s'enfoncent profondément dans la roche massive et leurs chantiers d'extraction montrent de grandes excavations et des fronts bien orthogonaux. Il ne s'agit plus ici d'artisans polyvalents mais bien de professionnels du génie, spécialistes de l'extraction: exemptores, lapicidinarii et quadratorii. Ceux-ci se bornaient à extraire et à équarrir sommairement les blocs, afin de faciliter leur transport sur un chantier de taille à pied d'œuvre, où ils étaient terminés ou épannelés.

Il est possible, sinon probable, que ces légionnaires se soient organisés à la manière des entreprises de travaux publics ou du bâtiment, qui envoyaient des équipes de carriers sur le terrain, selon les besoins de la construction. Le terme de vexillatio, utilisé à diverses reprises sur les documents épigraphiques, illustre, au demeurant, le concept de détachement temporaire spécialisé dans une tâche déterminée, militaire ou civile. En cela, ces exploitations rappellent le fonctionnement des chantiers d'extraction du Bois des Lens et du Pont du Gard, où chacune des phases d'extraction était étroitement liée à une grande commande précise, et non à un approvisionnement indéfini ou à un stockage commercial. Lorsque d'autres marchés de construction se présentaient, parfois quelques années après l'activité précédente, soit les carrières civiles initiales étaient déblayées en partie, soit on en ouvrait de nouvelles dans le voisinage immédiat, et ce n'était probablement pas les mêmes escouades qui les exploitaient. Le nombre des carriers qui composaient une telle équipe et la durée de leur présence dans un chantier d'extraction ont pu être évalués à une dizaine d'hommes et à une année en moyenne (Bessac, 1996, p. 314).

Une même carrière pouvait connaître, dans le temps, des phases différentes en matière de structure d'exploitation. Ainsi, de grandes carrières, un temps exploitées par l'armée ou par des équipes de spécialistes de l'extraction et chargées de fournir un important chantier de construction, ont été réoccupées ensuite par un ou plusieurs artisans assurant une plus large part de la chaîne de production, ou sa totalitć. C'cst cc qui scmble s'être passé dans les carrières de Locuon, de La Lie et surtout de Saint-Boil. Dans celle-ci, l'exploitation de blocs de grand appareil du début du Haut-Empire a laissé ensuite la place à des activités plus artisanales, notamment à des carriers/sculpteurs qui devaient fonctionner un peu selon les mêmes schémas économiques que leurs collègues des sommets vosgiens, bien que leur production fût nettement plus « romanisée ». 
Cette évolution économique semble encore plus évidente avec la production de sarcophages. S'il n'est pas illégitime de restituer dans une carrière de pierre de taille, liée aux grandes constructions monumentales, des produits destinés à quelques sculpteurs, qui s'approvisionnaient en marge de l'activité principale, il est plus difficile d'imaginer le même schéma avec une production de sarcophages, en particulier dans des chantiers souterrains. Dans la carrière de La Lie, il est clair qu'il existait une relative systématisation de l'extraction des sarcophages. Les couvercles étaient extraits verticalement alors que les cuves l'étaient horizontalement, dans le sens de la stratification. La systématisation de cette production, même si elle restait approximative, aurait mal supporté un mélange avec l'extraction de modules de blocs pour la construction, qui aurait désorganisé toute l'exploitation. Il est vrai que le développement de l'inhumation en sarcophages, à partir du III' s., a coïncidé avec une réduction très sensible, pour ne pas dire un arrêt, de la construction en pierre de taille. Les carrières romaines initiales, ouvertes à ciel ouvert pour la plupart, ont connu alors des changements radicaux. Plutôt que de les étendre en surface ou en profondeur, les carriers de l'Antiquité tardive et de l'époque mérovingienne ont préféré généralement progresser en souterrain en partant de la base des fronts anciens. Cette stratégie a été adoptée à La Lie, et aussi ailleurs, notamment à Seyssel, sur le Rhône (Dufournet, 1976), ou dans l'Yonne (Poulain, 1954). Elle évitait la suppression de la découverte, cette masse de matériau inutilisable qui recouvre d'habitude la roche saine. Il existait cependant des exceptions à ce schéma, comme les carrières du mont Béout, près de Lourdes, qui, de l'Antiquité tardive à la période mérovingienne, constituèrent de nouvelles ouvertures, généralement limitées, pour des raisons d'économie de travail et de difficulté de transport, à un seul niveau d'exploitation (cf. Boudartchouk, supra, p. 53-60). Les fabricants de sarcophages assuraient aussi le creusement des cuves, ne serait-ce qu'afin de les alléger avant leur transport. Là encore, les carriers du mont Béout constituent une exception, puisque les auges n'étaient pas évidées sur place. Le phénomène trouve sans doute son explication dans les contraintes du relief, qui rendaient l'opération difficile in situ. On ne sait pratiquement rien du statut de ces fabricants de sarcophages ni de leur organisation économique, malgré la fouille de deux chantiers spécialisés : à La Lie et à Saint-Boil. Dans la carrière de La Lie, l'excellente homogénéité des petits groupes d'empreintes d'extraction permet de supposer l'activité de quelques artisans qui devaient s'entraider dans les tâches de bardage nécessitant plusieurs personnes. L'hypothèse de grandes équipes de fabrication de sarcophages, à l'image des carriers précédents, est difficilement admissible dans le contexte de l'époque. Même l'ampleur des travaux du mont Béout paraît plus liée à la multiplication de petits ateliers qu'à une entreprise globale d'envergure. Par rapport aux artistes sculpteurs, la grande différence de ces nouveaux spécialistes résidait surtout dans le fait qu'ils monopolisaient le chantier d'extraction. Ils se distinguaient aussi des équipes de carriers liées à la construction parce que, contrairement à eux, ils n'étaient pas itinérants mais vraisemblablement rattachés à une carrière.

Dans le lot des carrières antiques qui sont présentées ici, deux exploitations d'ampleur très différente révèlent néanmoins des points communs en matière économique et chronologique : la carrière de L'Estel, qui a fonctionné au milieu du I ${ }^{\text {er }}$ s. dans le sud de la Gaule, et celle du Tendu, ouverte quelque temps auparavant dans le Centre. Toutes deux ont été exploitées en bordure d'une rivière et ont produit un matériau assez commun, qui a été utilisé dans la construction d'un ouvrage d'art. Leur fonctionnement antique a donc été assez bref et elles n'ont connu que très tardivement une reprise de leur activité. Dans les deux cas, leur dépendance de la voie d'eau pour le transport de leur production est évidente. Toutefois, seul, le chantier de L'Estel a subi des contraintes hydrauliques directes en période de hautes eaux, qui ont obligé ses exploitants à adopter une stratégic d'exploitation très spécifique. Ces deux sites montrent donc des aspects de l'exploitation romaine tout à fait inédits jusqu'ici. Les seules carrières de Gaule déjà connues pour avoir été exploitées au ras de l'eau sont celles de La Couronne, qui ont fonctionné dès l'époque grecque pour la ville de Marseille; malheureusement, leurs activités postérieures ont un peu faussé leur aspect antique (Trousset, Guéry, 1981 ; Guéry et al., 1985). Les carrières de marbre de Saint-Béat étaient, elles aussi, installées à quelques dizaines de mètres seulement de la Garonne, mais aucune trace archéologique de l'utilisation de la rivière pour le transport n'a pu être mise en évidence, malgré une prospection serréc des lieux, et le seul argument épi- 
graphique disponible est sujet à caution (Fabre, Sablayrolles, 1995, p. 150-151).

Dans leur ensemble, les carrières antiques du Bois des Lens près de Nîmes produisaient du grand appareil, qui était surtout destiné au riche décor architectural et à la sculpture. Bien qu'il ne fasse pas partie des roches marbrières, on peut considérer ce matériau comme une sorte de succédané de marbre blanc. Son exploitation, durant le début du Haut-Empire, a donc adopté certains schémas comparables, notamment dans la diffusion de la production. Malgré l'éloignement des zones de production de toute voie navigable, des équipes de carriers y venaient pour extraire des blocs de plusieurs tonnes, qui étaient expédiés, seulement équarris, ou sommairement arrondis pour les éléments de colonnes, sur des chantiers de construction assez lointains, comme Nice ou Narbonne. L'étude analytique des traces montre clairement que, contrairement à la carrière de L'Estel, dont la production était dominée par des grandes séries de blocs modulaires, les carricrs du Bois des Lens disposaient des mesures exactes de chaque bloc de la commande, même s'il existait également de petites séries modulaires. Ces différents formats de blocs étaient répartis sur les sols de carrière, insérés au mieux dans la trame des fissures et des autres particularités géologiques de la roche. Ce mode de fonctionnement s'observe également dans plusieurs autres chantiers d'extraction, qui ont connu une activité durant la première moitié du Haut-Empire : La Lie, Saint-Boil et probablement Locuon, bien que, pour ce dernier, la recherche sur le terrain n'en soit qu'à ses débuts et qu'il manque encore des éléments pour asseoir une certitude. Ce type d'exploitation à la commande, avec des équipes différentes, qui ne demeuraient que de quelques mois à un ou deux ans sur place, favorisait une gestion soumise aux seuls intérêts du chantier de construction, et non à ceux de la carrière. Celle-ci n'était donc gérée qu'à très court terme, et cela se matérialisait essentiellement par trois phénomènes :

- une extraction en fosse afin d'èviter les investissements de creusement d'accès latéraux et les enlèvements inutiles de découverte sur des surfaces importantes;

- un comblement rapide des chantiers, en fin de commande, par accumulation des déchets à proximité immédiate des fronts de taille, à l'intérieur des excavations ;

- l'ouverture de plusieurs excavations côte à côte, qui pouvait résulter du fonctionnement simultané de plusieurs équipes indépendantes, mais aussi, et surtout, des trop grands frais qu'il aurait fallu engager pour évacuer tous les déchets des chantiers antérieurs.

\section{LES CARRIERS}

L'absence d'une base de données suffisante en matière de vestiges d'habitat, conséquence de découvertes trop peu nombreuses et de fouilles en extension trop rares, rend difficile, pour ne pas dire illusoire, une approche des carriers eux-mêmes, de leur origine, de leur statut, de leur organisation, de leur culture matérielle et de leur vie quotidienne. Faute d'avoir étudié suffisamment leurs lieux de vie, on ne dispose que de très peu d'indices pour répondre à ces questions.

Pour les premiers carriers des régions côtières de la Méditerranée, les rares informations que l'on possède, sur la nature des cultes qu'ils ont introduits en milieu indigène dans la région nîmoise, sur les décors architecturaux des monuments locaux construits avec leur production de pierre, inciteraient à leur attribuer une origine orientale (Bessac, 1996, p. 296-297). On ne saurait, cependant, généraliser : dans le domaine des métiers, comme dans bien d'autres, la situation de la Narbonnaise parait distincte de celle des autres régions de la Gaule (Béal, 2000, p. 149-172). Dans les Pyrénées, l'analyse des sources épigraphiques et des cultes ne décèle qu'une influence latine, soulignée par l'acculturation de la population locale libre, dont la participation à l'activité extractive des marbrières de Saint-Béat est évidente (Fabre, Sablayrolles, 1995, p. 150-156). La confrontation des deux exemples suggère donc deux phases: des premiers temps marqués par la présence d'une main-d'œuvre étrangère, qui diffuse sa culture autant que son savoir-faire; puis une période d'acculturation de populations locales à la technique extractive et de transformation comme à des pratiques matérielles, intellectuelles ou religieuses dans sa vie quotidienne. De l'acquisition du savoir-faire pourrait témoigner l'observation d'une prédominance de gauchers dans certaines carrières du Nord-Est (Röder, 1957, p. 260-261) et du Sud (Bessac, 1996, p. 296) : ce constat suggère l'existence de contraintes d'apprentissage technique, spécifiques au métier de carrier romain, et absentes chez. leurs prédécesseurs. L'évolution ne saurait cependant se réduire à un schéma unique. Ainsi, s'il n'est pas toujours aisé de 
distinguer, dans les marmorarii pyrénéens du $\operatorname{II}^{*}$ s. de notre ère, les autochtones de spécialistes venus d'ailleurs, il ne fait pas de doute qu'il existait des différences culturelles et techniques assez. fortes entre les carriers légionnaires du Nord-Est (cf. Lukas, supra, p. 155-174) et leurs collègues et voisins, les artisans/sculpteurs des sommets vosgiens (cf. Heckenbenner, Meyer, supra, p. 145-154). Par rapport aux militaires romains, ces carriers en milieu indigène occupaient une place comparable à leurs confrères protohistoriques de l'arrière-pays méditerranéen face aux équipes très structurées qui exploitaient les carrières côtières de Marseille. Ils leur empruntaient certaines pratiques techniques et quelques bribes de stratégie générale d'exploitation, mais ils restaient dans un système de production de tradition indigène.

Tout aussi délicate, en raison de l'absence de sources, est l'analyse des conditions juridiques des carriers. Quelle que soit l'origine de ces spécialistes de l'extraction des pierres de construction, on peut supposer qu'ils appartenaient à une profession d'itinérants assez corporative, au moins à ses débuts en Gaule. Aucune des recherches récentes n'a confirmé l'hypothèse de grandes troupes de condamnés ou d'esclaves carriers, comme on en signale dans les exploitations impériales ${ }^{108}$ (Dubois, 1908, p. 36-37). L'absence de ce type de carrière en Gaule est peut-être la cause de cette lacune. Les seuls documents utilisables dans ce domaine d'étude sont les inscriptions de la carrière de marbre blanc de Rapp, à Saint-Béat, et celles des vexillationes de légionnaires du Nord-Est (Fabre, Sablayrolles, supra, p. 61-81 et Lukas, supra, p. 155-174). Elles révèlent des situations bien différentes : celle de soldats légionnaires, donc citoyens, temporairement détachés de leur corps, et sans doute choisis pour leurs compétences, et celle de pérégrins libres pyrénéens, largement dominants dans le corpus des inscriptions de Saint-Béat, où ne figurent qu'un esclave et un affranchi (Fabre, Sablayrolles, 1995, p. 154-155). Malgré le peu de représentativité des données disponibles, elles n'en mettent pas moins en évidence des situations sans doute contrastées, où, malgré tout, la population libre, citoyenne ou pérégrine, paraît tenir une place considérable. À ces différenciations juridiques,

108. L'hypothèse selon laquelle des carriers auraient été attachés à des anneaux, découverts paraît-il dans leurs habitations des carrières de porphyre, à Boulouris-sur-Mer dans le Var, est peu crédible (léger, 1875 , p. 705) ; une mauraise interprétation de vestiges d'écuries semble plus vraisemblable. dont il ne subsiste que de rares traces épigraphiques, se superposaient probablement des discriminations techniques et professionnelles. Les techniques d'extraction font en effet appel à un savoir professionnel totalement différent selon qu'il s'agit de marbre ou de calcaire tendre, par exemple. Il est donc légitime de s'interroger sur l'existence de catégories spécialisées dans l'extraction de telle ou telle roche ou dans telle ou telle phase du processus. La question est, pour l'heure, sans réponse, en l'absence à peu près totale de données : les sources épigraphiques gauloises sont trop peu nombreuses en la matière et sont limitées, une fois encore, aux exploitations marbrières des Pyrénées et aux chantiers légionnaires du Nord-Est.

Ces situations contrastées d'origine géographique, de statut juridique et, peut-être, de compétences techniques pouvaient se rencontrer au sein même des exploitations ou dans des exploitations suffisamment proches pour que des mutations techniques ou culturelles, nées de l'échange, se fassent jour. Un vestige mobilier fréquemment retrouvé en liaison avec l'activité des carrières constitue, dans ce domaine, un intéressant élément d'évaluation : il s'agit des autels votifs. Particulièrement nombreux dans la haute vallée de la Garonne (cf. Fabre, Sablayrolles, supra, p. 61-81), où ils ornaient non seulement le sanctuaire découvert dans la carrière de marbre de Rapp, mais aussi tous les sanctuaires d'altitude ou de communautés dédiés aux divinités topiques pyrénéennes, ils se retrouvent dans l'environnement de la plupart des carrières. Cette production a ainsi été également reconnue dans les exploitations du Bois des Lens, où elle constitue le seul type d'œuvre élaborée issu des chantiers d'extraction de la pierre (Bessac, 1996, p. 31-32 et 67-68). Dans la carrière de Saint-Boil, la production d'éléments cultuels est attestée, elle n'est cependant pas aussi caractéristique, car elle prend place au milieu d'autres ouvrages plus complexes. La carrière de granite de Locuon a également produit des autels votifs, comme en témoignent les réemplois dans l'église qui surplombe l'excavation. Il en est également signalé dans les exploitations du Nord-Est. Si certaines productions pyrénéennes, parfois de grande taille, présentent une facture élaborée, avec des moulures et des décors soignés, des traditions d'atelier, la majorité de ces autels est taillée dans des déchets d'extraction inutilisables comme pierre de taille. D'une manière générale, leur taille sommaire, leur forme à peine ébauchée, la mal- 
adresse des rares décors figurés prouvent que leur fabrication n'était pas le fait de tailleurs de pierre très chevronnés. Il s'agissait plutôt de l'œuvre de carriers, tailleurs occasionnels, qui ne maîtrisaient guère la technique de dessin et de façonnage des moulures, mais qui possédaient quelques notions de taille, ne serait-ce que par les contacts qu'ils avaient avec les spécialistes. La diffusion de ces pièces dans l'environnement régional, bien marquée dans le haut bassin de la Garonne, suggère une commercialisation locale, à destination d'une population peu aisée et en voie de romanisation, qui trouvait dans ces objets sommaires et donc, sans doute, bon marché un moyen à sa portée de manifester son adhésion aux modes de la culture dominante. Pour le carrier, tailleur occasionnel, ces modestes productions pouvaient constituer une source parallèle de revenus. Ces autels sont absents d'une grande carrière : L'Estel, qui a fourni des pierres pour les ouvrages d'art de l'aqueduc de Nîmes, surtout pour le plus grand, le Pont du Gard. Dans ce chantier, il y avait certainement d'excellents spécialistes de l'extraction, mais leur savoir professionnel s'arrêtait probablement à la production en série de grands blocs modulaires ; la position de la carrière, en zone inondable, et l'organisation générale du chantier de construction, très proche, ne laissaient peut-être ni la place ni le loisir aux carriers de produire en parallèle des objets cultuels.

Les productions cultuelles, tout à fait marginales d'un point de vue économique dans la carrière, n'en ont cependant pas moins contribué à la diffusion de nouvelles formes de dévotion et peut-être même, plus profondément, à la transformation de la pensée religieuse, comme l'ont souligné J.-M. Fabre et R. Sablayrolles dans leur contribution (p. 61-81). En l'état de la recherche, il semble qu'en plusieurs endroits les carriers romains aient introduit, en plus de leurs techniques, des cultes nouveaux qui ont parfois modifié des cultes indigènes préexistants. Ce constat est un argument complémentaire à l'onomastique pour démontrer la venue de spécialistes étrangers au moment du démarrage des exploitations. Nul n'en sera surpris, en particulier dans le Nord-Est, où les légions prirent au moins partiellement en charge cette activité, et dans les zones d'extraction nouvelles, à l'exemple de celle du granite de Bretagne ou des marbres dans les Pyrénées. On pourrait s'en étonner, en revanche, dans les bassins carriers de la bordure méditerranéenne, comme celui du
Bois des Lens, où une activité extractive antérieure à la période impériale est maintenant clairement attestée, y compris dans l'arrière-pays. Dans cette région, les équipes de carriers de tradition hellénistique ont certainement côtoyé les professionnels romains pendant plusieurs décennies après la romanisation matérielle des métiers de la pierre, que l'on peut situer aux environs des années 30 avant notre ère (Bessac, 1988a). Mais ces deux groupes de carriers appartenaient à des systèmes culturels et économiques suffisamment différents pour qu'une fusion rapide soit impossible. Il en allait de même pour les tailleurs de pierre et les constructeurs en général (Bessac, Fiches, 1979, p. 150). Dans le domaine culturel, le rôle des carrières et des carriers comme vecteurs de pratiques nouvelles ne fut donc pas uniforme et, comme dans le cadre des origines, des statuts juridiques et des structures d'exploitation, les sources indiquent plutôt des situations contrastées.

\section{L'OUTILLAGE ET LES TECHNIQUES}

\section{L'EXTRACTION}

Jusqu'à une époque récente, les publications qui présentaient des outils d'extraction souffraient d'une information archéologique insuffisante. Les outils avaient été souvent recueillis au cours de déblaiements d'anciennes excavations, sans que fût analysée leur position stratigraphique ; il était, dès lors, impossible de les rattacher à une des phases d'activité de chantiers qui avaient parfois fonctionné depuis l'Antiquité jusqu'à l'époque moderne. Certains de ces spécimens provenaient des anciennes collections de musées et leur datation ainsi que leur provenance étaient encore plus difficiles à préciser. Le contexte archéologique des découvertes, très mal défini, ne permettait pas de savoir si ces outils servaient à l'extraction, au débitage ou à la taille. Or, selon qu'il est employé à mème le rocher, pour le creuser, ou sur un bloc déjà extrait, pour l'équarrir sommairement, ou au stade de la taille, un pic, par exemple, n'a ni le même profil ni le même poids. Le problème se pose en des termes analogues pour plusieurs autres outils, en particulier les coins.

Les récentes recherches en carrière ont amélioré la situation, surtout grâce aux nouvelles découvertes d'exemplaires bien datés et parfois abandonnés, ou 
oubliés, sur le lieu de leur emploi, voire en position de travail (cf. Bessac, supra, p. 29-51). Pour les traces d'outils, seules les plus évidentes (saignées d'encoignure, trous d'emboîture, tranchées de havage) avaient été parfois décrites en détail, mais sans jamais de véritable garantie chronologique. À la notable exception près des observations de J. Röder, les impacts d'outils sur les fronts et les sols de carrière n'avaient jamais été analysés dans la perspective d'une confrontation avec les instruments issus des carrières, et encore moins dans l'espoir de retrouver le geste exact du carrier. Les études actuelles commencent à pallier certaines de ces carences et rattrapent ainsi une partie du retard pris dans cette forme d'approche, que les préhistoriens pratiquent déjà depuis longtemps dans l'étude des silex. Il reste néanmoins quelques zones d'ombre et d'incertitudes dans les interprétations de ces traces d'extraction. En l'état actuel de la recherche, il est donc plus prudent de considérer comme sûrs les seuls témoignages pour lesquels on peut associer concrètement l'outil et sa trace. Les hypothèses fondées sur l'interprétation des traces ne sont validées que lorsqu'elles peuvent être vérifiées par l'archéologic expérimentale ou par l'observation ethnographique directe ${ }^{109}$.

Les nouvelles découvertes d'outils d'extraction concernent en premier lieu les coins de fer. Plusieurs exemplaires ont été mis au jour, dans les carrières de grès des Vosges, à La Croix-Guillaume, dans celles du sud de la Gaule, ouvertes dans le calcaire fin des Lens et dans la mollasse coquillière de L'Estel ${ }^{110}$. Dans la carrière de La Lie, des emboîtures du Haut-Empire et des encoignures de l'Antiquité tardive et d'époque mérovingienne portent des traces très caractéristiques de la pression métallique des coins. Des traces analogues existent dans la carrière du Tendu et dans celle de Locuon, mais leur datation n'a pu être précisée. Sur le seul site de Saint-Boil s'est posée la question de la matière de l'outil. L'archéologue qui a fouillé la carrière a découvert, dans

109. Au cours des enquêtes ethno-archéologiques que nous avons conduites auprès d'anciens carriers, il est parfois arrivé qu'ils attribuent à leurs prédécesseurs l'usage d'une technique qu'ils n'avaient connue que par les livres; le caractère irréfutable de l'écrit pour un travailleur manuel peut donc conduire à des témoignages faussés.

110. Selon leur degré d'usure, leur longucur varie de 13 à $20 \mathrm{~cm}$ environ ; ils sont amincis sur leurs deux tiers, mais ne sont généralement pas tranchants; le tiers restant est de section rectangulaire $(5,5 \mathrm{~cm} \times 3,5 \mathrm{~cm}$ en moyennc) avec des chanfreins sur ses arêtes; l'angle de ces coins est d'environ $15^{\circ}$. les déchets d'extraction, des empreintes de coins de bois ; il a identifié, par ailleurs, de la poussière ligneuse dans une emboîture du Haut-Empire. Sur ce problème, l'excellente mise au point de A. Dworakowska au sujet de l'usage des coins de bois dans l'Antiquité incite à la prudence face à une telle interprétation des traces de coins (Dworakowska, 1988). L es témoignages qu'elle a recueillis de professionnels montrent que les emboîtures destinées à cet usage étaient tronconiques, afin de recevoir des coins de bois assez volumineux, dont la dilatation, sous l'effet de l'eau, exerçait une pression suffisamment forte pour obtenir la fracture de la pierre. Il semble que ce ne soit pas le cas à Saint-Boil où les embồtures sont de section angulaire. La carrière de Saint-Boil paraît donc constituer une exception parmi les carrières romaines, où l'emploi de coins en fer est la règle générale. L'usage du bois paraissait limité à de fines cales (appelées paumelles par les carriers modernes), qui étaient placées de chaque côté du coin de fer pour faciliter la répartition de la pression de l'outil sans faire éclater la pierre (Bessac, 1996, p. 202). Des coins de bois taillés en sifflet étaient également utilisés en carrière, mais pour caler les blocs extraits lors du bardage, du débitage et de la taille. L'usage des coins de bois pour l'extraction des pierres de Saint-Boil est donc, en l'état actuel des données, un unicum dans les carrières d'époque romaine.

Le deuxième outil, le pic d'extraction, sert à creuser des tranchées de havage, larges, en fonction de leur profondeur, de 7 à $15 \mathrm{~cm}$. Le pic peut se présenter sous diverses formes, dont la plus commune est l'escoude. Il en est fréquemment question dans les commentaires sur les traces d'outil, toutefois sa découverte est fort rare et il n'y a guère que la carrière Héral-Nègre, dans Le Bois des Lens, qui en ait fourni un exemplaire entier. Plusieurs autres fragments de ce type d'outil ont été découverts dans le même affleurement, à l'intérieur de la carrière de Mathieu, et ont pu être mis en relation directe avec leurs traces. L'outil est surtout caractérisé par la grande longueur de son fer $(0,50 \mathrm{~m}$ environ) et par ses extrémités actives, munies d'une double dent. Ces particularités ont permis d'identifier ses impacts dans plusieurs exploitations de Gaule, notamment dans la partie la plus ancienne de La Lie, dans L'Estel et à Crazannes en Charente-Maritime (Bocquet, Valat, 1995, p. 50-51). Dans les carrières de Rhénanie, sa présence semble attestée par un graffito de carrier romain (Röder, 1957, fig. 21-1), 
mais des expertises restent à réaliser sur des fronts rocheux de roches non abrasives. Il existe aussi des formes d'escoudes à tranchant droit, disposé perpendiculairement au manche de l'outil. Elles ne sont connues que par leurs impacts sur les pierres tendres et fermes, mais les tranchées de havage qu'elles ont servi à creuser sont tout à fait comparables à celles que creuse l'escoude à double dent. On peut donc supposer pour les deux modèles une forme générale assez proche. Ce dernier type d'escoude est le plus ancien et semble avoir été introduit en Gaule méditerranéenne par les colons grecs (Bessac, 1988a, p. 61-62) ; il disparaît de cette région dès le début du Haut-Empire, pour réapparaître à l'Antiquité tardive. La chronologie précise de son usage dans la Gaule septentrionale reste à préciser, voire à établir. Dans les carrières de La Lie, cettc version de l'outil succède à celle de l'escoude à double dent, à l'instar de ce qui se passe dans le Sud. L'interprétation des mêmes traces par G. Monthel, dans le calcaire tendre de Saint-Boil, lui fait toutefois supposer un outil très étroit, à bords parallèles et à manche fin et souple. Cette hypothèse d'une variante de l'escoude ne pourra être confirmée que par la découverte d'un tel outil dans un contexte archéologique sûr. Dans le granite de Locuon, de même que dans le grès des Vosges, matériaux plus résistants et plus abrasifs que les calcaires évoqués ci-dessus, une forme de pic d'extraction à pointe pyramidale a été employée. S'agit-il vraiment d'une escoude comparable aux modèles découverts dans Le Bois des Lens ou au graffito de Rhénanie? On ne peut le déterminer avec certitude en l'absence de découverte d'une forme complète. Des modèles de pics d'extraction à profil en feuille de laurier, à l'image des outils mis au jour en Allemagne (Sprater, 1948, p. 42), ont pu être également employés dans ces tâches.

D'autres outils ont été utilisés dans l'extraction antique, en particulier des mortaisoirs, qui ont servi à creuser les emboîtures et les encoignures d'époque romaine. Bien qu'aucun spécimen de cet outil n'ait été découvert, ses traces très caractéristiques, identifiées sur les calcaires de Gaule méditerranéenne, attestent un modèle à tranchant droit et perpendiculaire au manche, propre à la période du Haut-Empire (cf. Bessac, supra, p. 29-51). Son usage ne semble pas attesté de manière certaine à la même période dans les exploitations plus septentrionales. Dans celles-ci, notamment à Saint-Boil, le creusement de trous et de saignées pour placer les coins était réalisé à l'aide d'un outil pointu. La même technique s'observe dans le grès des Vosges, à La CroixGuillaume, et dans le granite de Locuon. Il est vrai que, dans le travail de ces roches abrasives, il était normal de préférer un modèle à pointe à un outil muni d'un tranchant.

Les carriers antiques utilisaient également, pour extraire les roches, la masse ou la mailloche, qui percutent les coins, la pince de carrier et les rouleaux, pour barder les blocs, la curette de nettoyage des tranchées de havage, les pioches et pelles pour supprimer la découverte, etc. Compte tenu du faible volume fouillé dans les carrières de Gaule, il est déjà statistiquement extraordinaire qu'un outil aussi volumineux et lourd qu'une escoude $(6,3 \mathrm{~kg})$ ait été découvert (cf. Bessac, supra, p. 29-51). L'oubli ou la perte, dans l'Antiquité, d'un tel instrument sont d'autant plus surprenants que toutes les pièces de fer, même lorsqu'il s'agissait d'objets usés, étaient systématiquement recyclées dans les forges de chantier, comme celles de la carrière de Mathieu dans Le Bois des Lens ou de la carrière de Saint-Boil. Le grand nombre d'outils découverts par F. Sprater dans les carrières du Kriemhildenstuhl et par J. Röder dans celles de l'Odenwald et de la Pellenz. n'en est que plus étonnant (Sprater, 1948, p. 42 ; Röder, 1957, p. 232). Il est certain que ces chercheurs ont déblayé les excavations plus qu'ils ne les ont fouillées, dans le sens archéologique du terme ; les volumes de déblais qu'ils ont dégagés devaient donc être supérieurs à ceux qui ont été observés par les archéologues actuels. S'il existait une explication historique à cette exceptionnelle richesse des carrières du Kriemhildenstuhl, de l'Odenwald et de la Pellenz, comme l'abandon soudain des lieux d'extraction et des outils qui s'y trouvaient, elle ne saurait, en l'absence de données archéologiques plus précises, que relever de l'anecdotique et aucune hypothèse solide ne pourrait, en l'état actuel des sources, être proposée.

\section{LE LEVAGE ET LE TRANSPORT}

Les appareils de levage, généralement en bois, ne laissent que peu de vestiges ou de traces dans les carrières ou sur les blocs. C'est surtout leur implantation au sol qui pouvait mouler des empreintes dans les déchets d'extraction, ou creuser des trous dans le rocher. De tels indices ont été identifiés dans les carrières de La Lie et de 
La Croix-Guillaume, sans que l'on puisse déterminer la nature précise des engins. Dans la carrière de Mathieu, dans Le Bois des Lens, les trous de l'engin de levage n'ont pu être retrouvés car, sur le seul emplacement où l'on situe leur installation, les carriers du XIX ${ }^{e}$ s. ont arasé le sol. L'aire de stockage des blocs et le point de départ de l'accès charretier ont été, par contre, identifiés, de chaque côté d'une petite éminence rocheuse aménagée en terrasse. Au-delà de l'aire de stockage, dans une tranchée de havage, l'usure caractéristique provoquée par une corde enroulée autour d'un axe prouve l'installation d'une sorte de cabestan, destiné à manouvrer l'engin de levage. La position de cet appareil montre qu'il servait à déplacer sa charge (les blocs de l'aire de stockage) d'un côté à l'autre du promontoire sur lequel il était juché, vers l'accès charretier. Il ne pouvait donc s'agir d'une chèvre. L'hypothèse de l'usage d'une variété de dikolos, qui est une sorte de portique muni de moufles et pouvant se rabattre d'un côté et de l'autre, sans entrave au milieu, est envisageable ici (Bessac, 1996, p. 292, fig. 179-180). Dans le site de L'Estel, au Pont du Gard, les carriers romains avaient repoussé à la fin du chantier l'exploitation d'une énorme masse rocheuse saine, afin de disposer d'un socle pour pouvoir placer sur un point haut une partie de leurs engins de levage. D'autres appareils étaient disposés en face, au sommet des fronts, comme en témoigne l'orientation des encoches qui étaient destinées, semble-t-il, au logement de crochets de préhension des blocs. Ces crochets devaient exercer une traction oblique vers le haut, afin de permettre le basculement des blocs hors de leur emplacement géologique, une fois qu'ils étaient séparés du substrat. L'identification de ces grandes encoches, qui n'ont aucun point commun avec les trous de louve, est totalement inédite. Par ailleurs, nous disposons désormais d'un nombre suffisant de blocs bruts d'extraction, stockés au sein des carrières ou même déjà arrivés sur les chantiers de taille ou de construction, pour constater que jamais un trou de louve ou de forcipes n'a été creusé dans la pierre, à ce stade de la production. Il faut donc abandonner les propositions iconographiques qui montraient de tels moyens de préhension en carrière (Bedon, 1984, fig. 33-39; Bessac, 1992, p. 424, fig. 10). Les élingues de corde étaient vraisemblablement le moyen de préhension le plus communément employé pour le levage dans les chantiers d'extraction qui le nécessitaient. Les excavations peu profondes, comme celles de Saint-Boil ou de La Croix-Guillaume, étaient pourvues de rampes d'accès constituées de déchets d'extraction, pour permettre de sortir leur production sans recourir à des engins de levage.

La connaissance du transport des blocs n'a progressé récemment que dans le domaine fluvial. Dans la carrière du Tendu, un canal a été fouillé et, dans celle de L'Estel, de très forts indices plaident en faveur d'un transport fluvial et de l'existence d'un chenal latéral. L'usage d'embarcations à fond plat ou de radeaux doit être envisagé dans les deux situations. Seule, la fouille de la carrière de L'Estel se poursuit actuellement et permettra peut-être d'obtenir de nouveaux vestiges dans ce domaine.

\section{OUTILLAGE ET APPAREILS DIVERS EN CARRIÈRE}

Les autres outils et appareils propres aux tâches habituelles des carriers gallo-romains se limitaient aux outils de débitage, de tournage et de taille dans les exploitations où la chaîne de production se poursuivait jusqu'à l'œuvre terminée. L'usage de la scie dentée dans le débitage de la pierre n'est prouvé que dans deux carrières de calcaire tendre à Saint-Boil et, dans une moindre mesure, à La Lie. Le tour a été utilisé à SaintBoil. Il a également servi à façonner le calcaire ferme oolithique de la carrière de Mathieu dans Le Bois des Lens. Dans les deux cas, les structures en bois, dont sont d'habitude constitués ces appareils, n'ont laissé aucune trace et l'on peut seulement, par déduction, montrer que le modèle de Saint-Boil était vertical. L'extrémité active de l'outil d'attaque du tour, le crochet, était forgée en arrondi dans les deux exemplaires de Saint-Boil et du Bois des Lens. À l'instar du sciage, le tournage pouvait être confié à du personnel faiblement qualifié, car le crochet était, d'ordinaire, guidé par un profil découpé selon la moulure à produire.

Dans le domaine des activités annexes à l'extraction, il faut souligner l'apport exceptionnel de la carrière de Saint-Boil, qui, outre de nombreuses traces d'outils de taille (pic, marteau taillant, ciseau, etc.), a donné des documents extrêmement rares sur la préparation et le déroulement de la taille ainsi que de la sculpture (épures, dessins, ébauches, comptes). Cette découverte est d'autant plus extraordinaire que ce type de vestiges n'existe généralement plus sur les chantiers de taille au 
pied des monuments. L'ébauche très sommaire d'une sculpture rupestre, mise au jour dans la carrière de La Lie, permet d'espérer des découvertes de même nature sur ce site.

\section{LES PERSPECTIVES DE RECHERCHE}

Le premier et principal constat du bilan qui vient d'être dressé est que la recherche de terrain sur les carrières antiques de Gaule n'en est qu'à ses débuts. La diversité des situations régionales et la variété des problématiques peuvent donner une impression de désorganisation de la discipline, surtout si on la confronte avec l'apparente rigueur de notre programmation archéologique nationale. Mais cette variété constitue, en réalité, la richesse du thème et ne fait que traduire sa jeunesse.

Certains axes forts de la recherche ont été seulement effleurés dans les contributions à ce dossier. Il en est ainsi des diverses catégories d'analyses pétrographiques, dont les archéologues attendent des critères sûrs d'identification, permettant de mettre en relation le produit fini, découvert sur des sites parfois très éloignés des lieux d'extraction, et les carrières, d'où était issu son matériau. La confrontation des expériences récentes sur le marbre a montré l'apport et les limites de cette voie (Cabanot $e t$ $a l$. éds, 1995). La première condition du succès de la démarche est la représentativité de l'échantillonnage qui est réalisé sur les sites potentiels d'extraction. Sa validité suppose un travail de terrain long et minutieux, dans la mesure où la base de données n'aura de sens que si toutes les carrières ont été échantillonnées et que si chacune d'elles l'a été en plusieurs points. L'expérience pyrénéenne a montré que cette condition nécessaire n'était pas toujours suffisante: les approches archéométriques sont multiples et aucune n'est, à elle seule, satisfaisante ; seule, la confrontation de diverses grilles et de diverses méthodes d'analyse apporte des résultats probants. Cie constat, quelque peu décourageant par l'ampleur de la tâche qu'il dessine et le caractère aléatoire des résultats, ne doit pas entraver la recherche. L'importance de l'enjeu est en effet de taille : les études de diffusion des produits ne seront vraiment crédibles que le jour où les identifications seront pleinement assurées. Pour nuancer cette perspective quelque peu pessimiste, il faut souligner que la situation des matériaux de luxe, comme le marbre ou les calcaires fins, est à distinguer de pierres plus communes, dont la diffusion est à moins longue portée et dont l'identification risque donc de moins prêter à confusion.

L'une des grandes voies de la recherche, encore très lacunaire, reste la prospection. L'expérience a démontré, dans les vallées pyrénéennes ou autour du Bois des Lens, qu'elle donnait de bons résultats, même si cela ne se traduisait pas forcément par un accroissement quantitatif des connaissances. Il ne fait pas de doute que les affleurements de pierre de taille inexplorés, du point de vue archéologique, sont toujours aussi nombreux. Une perspective prometteuse en la matière peut être tracée à partir de l'évidence, rappelée dans la présente conclusion, de l'existence de carrières à proximité de tous les grands chantiers urbains de l'Antiquité, quand les conditions géologiques s'y prêtaient. La perspective est d'autant plus riche que ces carrières de matériau de construction n'ont pas forcément été réutilisées de manière systématique, comme peuvent l'être les carrières de pierres ornementales. La prospection pourra être d'autant plus fructueuse que les progrès récents accomplis en matière de typologie chronologique des techniques d'extraction, notamment dans le Sud-Est, permettent parfois de dater, sans recours à la fouille, certains fronts ou sols de carrière. Cette méthode ne saurait cependant s'appliquer de façon systématique, mais uniquement à l'intérieur d'un cadre régional, dont le sens historique et technique serait bien déterminé. Les potentialités incontestables de la prospection, que soulignent les résultats de ces dernières décennies, permettent d'envisager, à terme, une base de référence statistiquement mieux assurée pour des analyses du type de celles qui ont été présentées dans ce dossier, et dont le caractère aléatoire a été à plusieurs reprises souligné.

Les diverses techniques d'extraction antiques de la pierre de taille ordinaire semblent maintenant assez bien identifiées, même s'il reste des zones d'ombre, notamment dans les marbres, et des outils qui ne sont connus que de manière hypothétique. Sur ce dernier point, les carrières du Nord-Est semblent les plus prometteuses: leur position géographique près des limites de l'Empire romain a pu créer des conditions historiques favorables à de brusques abandons de chantier, même si l'historiographie récente invite, avec raison, à relativiser l'impact de ce qui était appelé naguère les invasions barbares. Des deux côtés du Rhin et même plus à l'est, il doit encore exister de bonnes opportunités archéo- 
logiques. La richesse des découvertes effectuées vers le milieu du siècle par F. Sprater et J. Röder ne peut qu'inciter à renouveler des expériences archéologiques, sur les bases désormais micux assurées de la stratigraphie et avec l'apport des recherches récentes sur le monde des carrières. La connaissance des diverses stratégies d'exploitation reste aussi à développer, surtout dans les grandes exploitations situées en bordure de rivière, où l'accès au moyen de transport constitue une contrainte et un atout, sur le plan technique comme sur le plan économique. La carrière de L'Estel est particulièrement bien placée pour cela, surtout si un lien peut être établi entre les données de la fouille actuelle et la rivière, par l'étude des vestiges d'aménagements hydrauliques antiques.

La fouille de carrière demeure donc une perspective essentielle de recherche, qu'elle soit rendue possible par les travaux de réexploitation moderne, par des projets d'aménagement de sites naturels, ou par une réflexion scientifique pertinente qui décèle dans le résultat de prospections le point fort méritant une fouille programmée. De nombreux aspects de la vie et des activités des exploitations antiques n'ont encore jamais pu être abordés de façon exhaustive, en particulier des questions aussi fondamentales que l'origine des spécialistes de l'extraction, leur condition sociale, leur mode de vie, leur intégration aux économies locales ou aux échanges lointains, leurs activités annexes. Les principaux ćléments de réponse à ces interrogations se trouvent dans la fouille d'habitats de carriers, que divers indices permettent de localiser approximativement dans les sites non fouillés (Bessac, 1986b, p. 161-163 et 1999a, p. 35-36). De ce point de vue, la recherche programmée constituera une incontestable priorité, dès qu'un site aux potentialités intéressantes sera dûment identifié.
Enfin la carrière doit être replacée dans son cadre économique, social et culturel, proche ou lointain. L'environnement immédiat des carrières, qui passe par une étude archéologique globale de la microrégion, peut permettre de mesurer l'impact de l'activité extractive sur la vie démographique, administrative, économique, sociale et culturelle des populations autochtones. Naturellement, les carrières de matériaux de luxe, qui ont produit sur la longue durée, constituent, de ce point de vue, un champ d'étude potentiellement plus riche que les carrières qui n'ont connu qu'un fonctionnement ponctuel ou sporadique. La diffusion du produit, à longue ou à courte portée, fait partie intégrante de cette analyse. Elle suppose un réexamen attentif, appuyé, le cas échéant, sur les progrès récents de l'archéométrie, des collections des musées et dépôts de fouilles, proches ou lointains. La validité de ce type de recherche repose sur l'exhaustivité du dépouillement et sur la rigueur des identifications, même si cette dernière précaution accroît, dans les statistiques, la proportion des laisséspour-compte. Là encore, matériaux de luxe et matériaux communs n'ont pas connu la même destinée, mais les uns comme les autres constituent des témoins importants de la vie économique antique et l'étude de la carrière ne saurait se limiter au seul espace du chantier. Les enquêtes entreprises dans ce sens autour du Bois des Lens, des bassins marbriers des Pyrénées, ou des sites vosgiens pourraient ainsi être étendues à d'autres ensembles de production de pierre.

Les directions de recherche ne manquent donc pas, les potentialités non plus et les richesses des acquis récents, dont témoignent le nombre et la variété des contributions ici rassemblées, constituent un point de départ, une base de données et une incitation à poursuivre l'œuvre engagée. 